\title{
Diseño de una Incubadora de Tecnología Intermedia Agrícola, Forestal y Turismo Rural
}

Design of an Incubator for Intermediate Agricultural Technology, Forestry and Rural Tourism

Contreras Márquez Araceli Janett ${ }^{1}$, Magaña Magaña José Eduardo ${ }^{2 凶}$, Villarreal Ramírez Víctor Hugo $^{2}$, Kiessling Davison Christian Mauricio ${ }^{2}$, Escalera Ochoa Laura ${ }^{2}$

${ }^{1}$ Estudiante de la maestría en Agronegocios, PNPC-CONACYT, ${ }^{2}$ Universidad Autónoma de Chihuahua-Facultad de Ciencias Agrícolas y Forestales

${ }^{凶}$ Autor de correspondencia: emagana@uach.mx

Recibido: $18 / 02 / 2017$

Aceptado: $30 / 06 / 2017$

\section{RESUMEN}

La Facultad de Ciencias Agrícolas y Forestales -Universidad Autónoma de Chihuahua tiene un Centro de Negocios, donde se atiende a emprendedores y empresas, involucrando a profesores y alumnos. No obstante, es necesario que este tenga reconocimiento oficial por la Secretaria de Economía Federal. Es por eso que se propone que este centro sea una Incubadora de tecnología intermedia agrícola, forestal y turismo rural. En nuestro país, el $80 \%$ de las empresas que no tienen un adecuado acompañamiento durante su formación, fracasan en el primer año de puesta en marcha, en tanto cuando hay un buen acompañamiento la cifra de éxito se eleva hasta un $93 \%$ de permanencia. Factores como, la inseguridad, pobreza, desigualdad, falta de oportunidades educativas, rezagos en el campo, son limitantes que frenan la actividad emprendedora. Para alcanzar este objetivo se desarrolló la metodología establecida por la Secretaría de Economía Federal, que comprende desde la organización en aspectos administrativos, características del proceso de incubación hasta la orientación a emprendedores. Los principales resultados fueron los manuales: Manual de la organización, estructura de la organización, de procedimientos e inducción a emprendedores. Se identificaron los servicios que se ofrecen de acuerdo a la planta de maestros y la infraestructura con que se cuenta. Entre ellos la elaboración de proyectos de inversión, estudios de mercado, corridas financieras, recursos humanos, elaboración de planes estratégicos y mercadotecnia. La conclusión final es que este tipo de Incubadoras promueven el emprendimiento de jóvenes estudiantes, profesores y productores agrícolas, forestales y turismo rural.

Palabras claves: Emprendedor, Incubadora, desarrollo de proyectos, integración, universidad y sector productivo, sector primario. 


\begin{abstract}
The Faculty of Agricultural and Forestry Sciences of Chihuahua has a business center where is given specialized care to entrepreneurs and businesses through various services, this involves teachers and students. However, it is necessary that this has official recognition by the Ministry of Economy. That's why it is proposed that this center is an incubator for forestry agricultural intermediate technology and rural tourism. In our country, $80 \%$ of companies that do not have adequate support during their training, they fail in the first year of implementation, while when there is a good accompaniment figure of success rises to $93 \%$ of permanence. Factors such as, insecurity, poverty, inequality, lack of educational opportunities, delays in the field are constraints that hinder entrepreneurial activity. To achieve this objective the methodology established by the Federal Ministry of Economy, ranging from the organization in administrative aspects, features the incubation process to targeting entrepreneurs was developed. The main results were the manuals: organization, organization structure, procedures and induction entrepreneurs the services offered according to the plant of teachers and infrastructure which has been identified. Including the development of investment projects, market research, financial runs, human resources, strategic plans and marketing. The final conclusion is that this type of Incubators promote entrepreneurship of young students, teachers and agricultural, forestry and rural tourism producers.
\end{abstract}

Key word: Entrepreneur Incubator, project development, integration, university and the productive sector, primary sector.

\section{INTRODUCCION}

Hablar de una incubadora de negocios es hablar de asesorías y orientación especializada para hacer realidad la idea de negocio de un emprendedor, aunque existen diferentes tipos de incubadora todas tiene el fin común, implementar todas las estrategias necesarias para llevar al éxito al emprendedor y su negocio. En el entorno económico actual, podemos ver que grandes compañías adoptan estrategias para reducir sus gastos y así poder incrementar su competitividad, esto ha llevado a las pequeñas y nuevas empresas que adoptar un rol importante en la creación de empleos y oportunidades de inversión, esto las ha llevado a convertirse en puntales en la economía. Para considerar la creación de empresas como una de las actividades que incrementan el desarrollo económico de un país, es necesario tomar en cuenta los factores que podrían influir en la formación de una nueva empresa o en el éxito o fracaso de la misma (García et al., 2004).

En nuestro país la creación de incubadoras de negocios ha sido progresiva, desde la aparición del concepto "incubadora de empresas" hasta nuestros días se han observado cambios y evoluciones que ayudan a que las empresas que se crean por este medio tengan más éxito y permanencia en el mercado. La tasa de éxito para las empresas que han sido gestadas en incubadoras oscila entre el $80 \%$ y el $93 \%$. En cambio, el $80 \%$ de las empresas innovadoras (no incubadas) cierran antes del año; entretanto, este número cae al $20 \%$ cuando se trata de empresas innovadoras graduadas en incubadoras 
(Marcano y García, 1996). El papel que desempeñan las incubadoras es determinante, ya que son fuente de conocimientos y asesoramiento para los empresarios y que les permitirá aumentar las posibilidades de éxito en el mercado. Las incubadoras evalúan ideas de negocio a la luz del mercado para que se generen innovaciones que permitan desarrollar un negocio de alto valor añadido, que pueda aportar desarrollo económico y social en su comunidad.

Cuadro 1: Servicios que proporcionan las incubadoras

\begin{tabular}{|c|c|c|}
\hline Tradicionales & $\begin{array}{l}\text { Tecnología } \\
\text { intermedia }\end{array}$ & $\begin{array}{l}\text { Alta } \\
\text { tecnología }\end{array}$ \\
\hline $\begin{array}{l}\text { Infraest } \\
\text { ructura física y } \\
\text { tecnológica. }\end{array}$ & $\begin{array}{l}\text { - Infraestruct } \\
\text { ura física y } \\
\text { tecnológica. }\end{array}$ & $\begin{array}{l}\text { - Co } \\
\text { nstitución de } \\
\text { empresas. }\end{array}$ \\
\hline $\begin{array}{l}\text { Servici } \\
\text { os para cubrir } \\
\text { requerimientos }\end{array}$ & $\begin{array}{lr}\text { - } & \text { Mecanismo } \\
\text { s de } & \text { operación } \\
\text { semiespecilizados. }\end{array}$ & $\begin{array}{l}\text { Me } \\
\text { cánica de } \\
\text { operaciones }\end{array}$ \\
\hline básicas. & $\begin{array}{l}\text { Procesos } \\
\text { semiespecializados, } \\
\text { incorporación } \\
\text { innovaciones. }\end{array}$ & $\begin{array}{l}\text { Ase } \\
\text { soría } \\
\text { especializada }\end{array}$ \\
\hline
\end{tabular}

Fuente: García, (2011)

En México la Secretaría de Economía (SE) a través del Sistema Nacional de Incubación de Empresas (SNIE) ha realizado una clasificación y estableció tres tipos de incubadoras de empresas: incubadora de negocios tradicionales, de tecnología intermedia e incubadoras de negocios de alta tecnología. En el país, las incubadoras están en su mayoría orientadas a atender empresas que trabajan sobre tecnología intermedia y negocios tradicionales, las incubadoras orientadas a empresas de alta tecnología representan $5 \%$, un porcentaje muy bajo del total de incubadoras en el país.

Objetivo general

Diseñar una incubadora de tecnología intermedia ofreciendo servicios profesionales, competitivos y dinámicos que garanticen estabilidad y permanencia en la creación y desarrollo de empresas innovadoras en los sectores agrícolas, forestales y de turismo rural.

Objetivos específicos

Establecer los lineamientos generales para la administración y operación de una incubadora de negocios de tecnología intermedia.

Identificar las características del proceso de incubación y los procedimientos a seguir de los proyectos incorporados a la incubadora.

Orientar a los emprendedores en el proceso de incubación.

\section{MATERIALES Y METODOS}

En el presente trabajo se propone la siguiente metodología: 


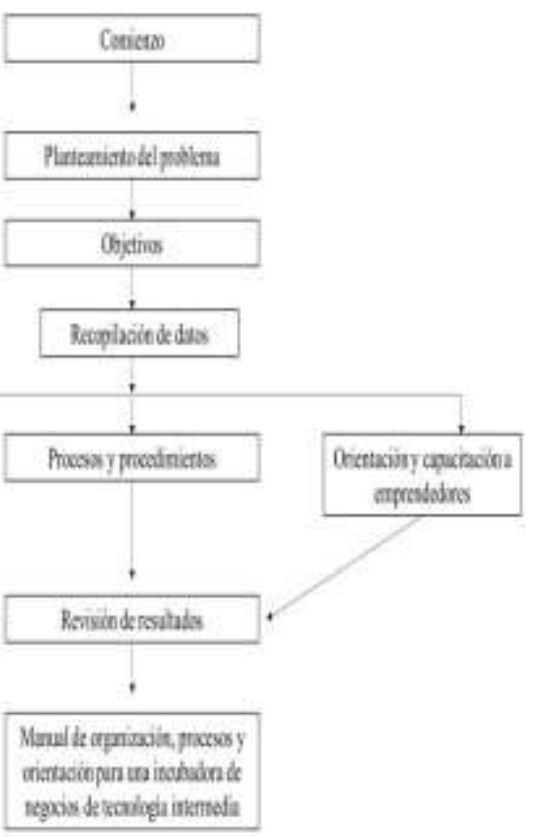

Figura 1: Metodología

\section{RESULTADOS Y DISCUSIONES}

Se elaboraron los objetivos generales y específicos para la incubadora propuesta.

\section{Objetivo General}

Impulsar y fomentar en los estudiantes, egresados y la sociedad en general el desarrollo de negocios en los sectores Agrícola, Forestal y Turismo Rural para contribuir al desarrollo económico de la región.

\section{Objetivos Específicos}

- $\quad$ Fomentar en los jóvenes, mujeres, empresaria(o)s y la sociedad en general la creatividad, innovación y espíritu emprendedor.

- Incrementar la formación de empresas competitivas de base tecnológica en los sectores agrícola, forestal y turismo rural.

- Formar empresas con proyectos innovadores y de valor agregado, que contribuyan al desarrollo económico y social de la comunidad.

- Reforzar a las empresas establecidas que tengan el propósito de desarrollar innovaciones tecnológicas en sus procesos, equipos y maquinaria.

- Proporcionar soluciones estratégicas al sector empresarial mediante capacitación y consultorías especializadas.

- Apoyar la creación, y registro de patentes, marcas y derechos de autor de productos innovadores.

Se diseñó la estructura organizacional de la incubadora, como se observa en la figura 1

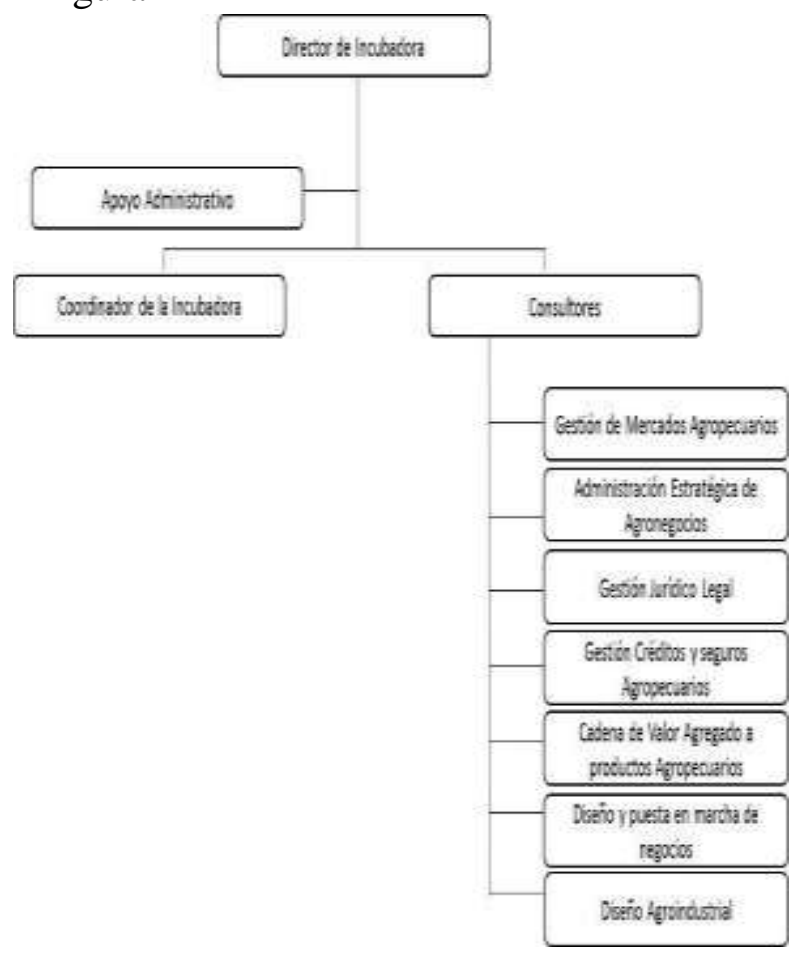

Figura 2: Organigrama de la incubadora Fuente: Secretaria de Economía, 2016

Servicios de la Incubadora de Negocios La incubadora además de las consultorías propias para cada proyecto incubado, ofrece a sus emprendedores y empresarios acompañamiento durante las etapas de su Modelo de Incubación, así como diversos servicios que los ayudarán a lograr la 
consolidación de sus ideas de negocio, recibiendo soporte integral a través de tutoría y asesoría especializada en áreas como administración, mercadotecnia y ventas, Fiscal, Contabilidad y finanzas, Gestión de exportación y manejo de aranceles, Manejo de aduanas, Cultura de calidad y marco normativo, Buenas prácticas agrícolas e inocuidad, Innovación y desarrollo tecnológico, Cuidado al medio ambiente, Capacitación empresarial, Enlaces de negocios, Vinculación con oportunidades de financiamiento e inversión Determinación del proceso de incubación para proyectos incorporados a la incubadora.

Modelo de Incubación de negocios

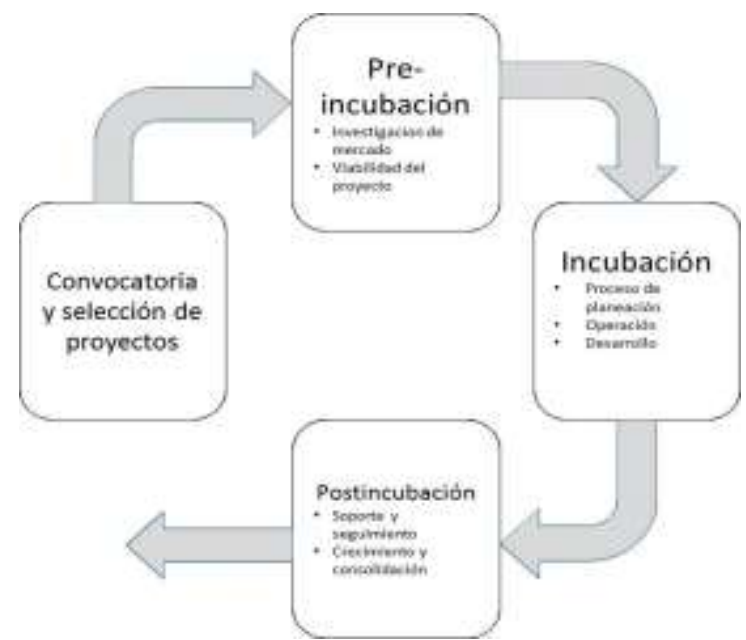

Figura 3: Modelo de incubación, para Incubadoras de Tecnología Intermedia Fuente: Secretaria de Economía, 2016.

Desarrollo del Modelo de Incubación de negocios Convocatoria y selección (23 días) Abarca desde el diseño y lanzamiento de la convocatoria hasta la selección de proyectos bajo las siguientes políticas:

a) La programación de los eventos de capacitación, selección y evaluación de proyectos dependerá directamente de las metas establecidas por la incubadora, así como las acciones que se determinen en la planeación para alcanzarlas.

b) No se pueden recibir proyectos, ideas de negocios o empresas con presencia en el mercado mayor a 1 año.

c) No se atenderán proyectos que ya haya recibido apoyo de otras instituciones de fomento al desarrollo emprendedor.

d) La incubadora determina el perfil de emprendedores con base en sus requerimientos.

e) El mínimo de consultores para la selección de proyectos será de 3, para asegurar la imparcialidad en la evaluación.

f) Los criterios de selección de proyectos serán:

a. Proyectos que den respuesta a una necesidad del mercado.

b. Proyectos que tengan identificado un mercado potencial.

c. Proyectos que sean susceptibles de registro de marca o patente.

d. Proyectos basados en la innovación o desarrollo en los sectores agrícola, forestal y turismo rural.

El proceso inicia con la publicación de una convocatoria que tendrá como finalidad la búsqueda y postulación de proyectos, esta será publicada por el Director de la Incubadora y estará dirigida para alumnos, exalumnos de la Facultad de Ciencias Agrícolas y Forestales y emprendedores externos a la misma.

En el proceso de selección participara el Comité Evaluador de Proyectos, para evaluar la factibilidad de cada proyecto y se tendrán tres tipos de respuesta a la postulación, las cuales serán: Proyecto aceptado, Proyecto condicionado y Proyecto rechazado.

1. Los proyectos aceptados seguirán a la siguiente etapa la Pre-incubación.

2. Los proyectos condicionados volverán a ser evaluados por el consejo 
técnico y dar una respuesta positiva o negativa.

3. A los proyectos rechazados por el consejo técnico se les evaluará su potencial si este resulta positivo se canalizará a otros programas de emprendedores, si su potencial resulta negativo se dará por terminado el proceso.

Pre-incubación (14 días) Esta etapa consta de cinco puntos clave; contrato de incubación, convenio de confidencialidad, diagnostico de necesidades del proyecto, plan de incubación y agenda de incubación, capacitación en desarrollo humano y empresarial.

Se imparten cursos básicos entre los cuales se proponen: Crecimiento Gerencial con Inteligencia Emocional, Plan de Vida y Trascendencia Empresarial, Mercadotecnia, Taller de

Propiedad Intelectual, Taller de Administración de la Producción y Taller de Finanzas.

Incubación (159 días) En esta etapa se propicia la integración de plan de negocios.

Se planea, organiza y controlan las actividades del proyecto en incubación con el propósito de que se inicie desde el estudio de mercado hasta el inicio de operaciones.

Es aquí donde se ofrece la capacitación y consultoría de las diferentes áreas como son: Gestión de mercados, Administración empresarial, Gestión jurídico-legal, Gestión financiera y fiscal, Procesos de producción, Desarrollo de imagen corporativa, Diseño industrial.
Post-incubación (261 días) Durante esta etapa, se brinda soporte y da seguimiento a la empresa con el fin de buscar su crecimiento y consolidación tanto en el mercado como en la sociedad en general. A lo largo de todo el proceso se brindan los servicios de tutoría y asesoría especializada por parte de todo nuestro equipo de expertos que integran la Incubadora.

Se da seguimiento a las operaciones una vez que la empresa lleva a cabo transacciones de manera formal, este seguimiento es mensual y se realiza en todas las áreas y adicionalmente cada trimestre se lleva a cabo la evaluación financiera de la empresa.

Actividades: Análisis mensual, Evaluación financiera trimestral, Liberación

Lineamientos para perfilar a posibles candidatos de la incubadora de negocios.

Inducción para Emprendedores

Con este documento se pretende que el participante de la Incubadora de Tecnología Intermedia de la Facultad de Ciencias Agrícolas y Forestales, identifiquen los puntos básicos en el proceso de incubación, ya que como hemos expuesto en puntos anteriores la capacitación y acompañamiento de expertos facilita el desarrollo y la puesta en marcha de ideas de emprendimiento innovadoras.

¿Quiénes pueden participar y que tipo de proyectos se incuban? Las incubadoras se interesan en ideas o proyectos con

potencial, teniendo elementos fundamentales como:

El mercado, tecnología, equipo, ventajas competitivas clave (factores críticos de éxito), visión enfoque en mercados abiertos, viabilidad atractiva e innovación. 


\section{CONCLUSIONES}

Se concluye que el proyecto es viable, ya que la Facultad de Ciencias Agrícolas y Forestales cuenta con instalaciones propicias, profesores especialistas en las áreas requeridas, y una amplia experiencia en el trato de los emprendedores. Como recomendación única es iniciar el proceso de Registro de esta Incubadora ante la Secretaría de Economía.

\section{LITERATURA CITADA}

García, C.; Martínez, A.; Fernández, R. (2004). "Análisis de los factores determinantes de la creación de empresas: Una evidencia empírica en Castilla y León”. Consejería de Economía y Empleo de la Junta de Castilla y León. España.

Hernández, P. P., \& Estrada, A. M. (2006). Análisis del sistema de incubación de empresas de base tecnológica de México. In Anais do Congreso Iberoamericana de Ciencia, Tecnología e Innovación.

Marcano, L., \& García, L. (1997). Las empresas de base tecnológica: opciones para la región. Revista Espacios, 18(2).

Secretaria de Economía (SE) 2015. www.economia.gob.mx

Instituto Nacional del Emprendedor (INADEM) 2015. www.inadem.gob.mx

http://www.inadem.gob.mx/incubadoras y_aceleradoras.html

http://www.inadem.gob.mx/docs/reglas/R eglas $\% 20 \mathrm{de} \% 200$ peracion $\% 20 \mathrm{del} \% 20 \mathrm{Fo}$ ndo $\% 20 \mathrm{PyME} \% 20-$

\%2028\%20Febrero\%202013.pdf 
Copyright (c) 2017 Araceli Janett Contreras Márquez, José E duardo Magaña Magaña,

Victor Hugo Villarreal Ramirez, Christian Mauricio Kiessling Davi son y Laura Escalera Ochoa

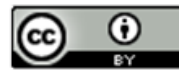

Este tex to está protegido por una licencia licencia Creative Commons 4.0

\begin{abstract}
Usted es libre para Compartir -copiar y redistribuir el material en cualquier medio o form ato- y Adaptar el documento -remezclar, transformar y crear a partir del material- para cualquier propósito, incluso para fines comerciales, siempre que cumpla la condición de:

Atribución: Usted debe dar crédito a la obra original de manera adecuada, proporcionar un enlace a la licencia, e indicar si se han realizado cambios. Puede hacerlo en cualquier form a razonable, pero no de form a tal que sugiera que tiene el apoyo del licenciante o 10 recibe por el uso que hace de la obra.

Resumendelicencia - Textocompletodelalicencia
\end{abstract}

